\title{
Partial Rank Modulation for Flash Memories
}

\author{
Zhiying Wang \\ Electrical Engineering Department \\ California Institute of Technology \\ Pasadena, CA 91125, USA \\ Email: zhiying@caltech.edu
}

\author{
Jehoshua Bruck \\ Electrical Engineering Department \\ California Institute of Technology \\ Pasadena, CA 91125, USA \\ Email: bruck@caltech.edu
}

\begin{abstract}
Rank modulation was recently proposed as an information representation for multilevel flash memories, using permutations or ranks of $n$ flash cells. The current decoding process finds the cell with the $i$-th highest charge level at iteration $i$, for $i=1,2, \ldots, n-1$. Motivated by the need to reduce the number of such iterations, we consider $k$-partial permutations, where only the highest $k$ cell levels are considered for information representation. We propose a generalization of Gray codes for $k$-partial permutations such that information is updated efficiently.
\end{abstract}

\section{INTRODUCTION}

Rank modulation was recently proposed as a robust and update-efficient information representation scheme for multilevel flash memories [1]. In rank modulation of $n$ flash cells, information is stored as the rank of the cells. Let $c_{1}, c_{2}, \ldots, c_{n}$ be the distinct analog values of electric charge in these $n$ cells and suppose $c_{p_{1}}>c_{p_{2}}>\cdots>c_{p_{n}}$. Then the induced rank vector is $\left(p_{1}, p_{2}, \ldots, p_{n}\right)$. For example, if $n=5$, $\left(c_{1}, \ldots, c_{5}\right)=(0.3,1.2,1.0,0.25,0.5)$, then the rank is $\left(p_{1}, \ldots, p_{5}\right)=(2,3,5,1,4)$.

In flash memory, to decrease the charge level of a cell, we have to first erase a complete block of cells (containing about $10^{5}$ cells [2]), which not only costs time and energy, but also reduces the lifetime of the device. In order to avoid overshooting when we increase the charge level, iterative injection is used in practice. The main advantage of using ranks over absolute values is that there is no need to charge the cells accurately. Each write operation only pushes one cell level to be the highest among the $n$ cells, so there is no risk of overshooting. We call this writing method push-to-thetop operation. Thus the writing process is accelerated and higher reliability is obtained. In addition, leakage (or deflation) causes charge loss among all cells simultaneously, though not evenly, which will facilitate more write operations.

Since information is represented by permutations of charge levels of the flash cells, the decoding process involves sorting of the levels - not a straightforward operation if implemented in hardware. The current proposal for a decoding architecture for rank modulation is based on an iterative process involving (1) maximal level identification: identifying the cell with the maximal level among the remaining cells and (2) candidates update: excluding the current maximal cell from future maximal level identification. Namely, for $n$ flash cells the decoding process has $n-1$ iterations.

Each iteration costs time, power, and circuit complexity. We would like to lower this cost while maintaining reasonable information capacity. Hence, we consider a generalized form of rank modulation, where information is represented by a permutation on the $k$ largest values out of the $n$ cells, for some $1 \leq k \leq n-1$. We call it $k$ partial rank modulation or $k$-partial permutation. The amount of information is $\log \left(\begin{array}{l}n \\ k\end{array}\right) k$ ! bits and decoding takes $k$ iterations. When $k=n-1$, it reduces to the original rank modulation. If we preserve the push-to-thetop write operations, we will get high speed and low error rate as in the original rank modulation. For example, when $n=5$ and $k=2$, we can represent $5 \times 4=20$ values while requiring 2 decoding iterations. On the other hand, when $k=n-1=4$, we can represent $5 !=120$ values with 4 iterations. The permutation $(2,3,5,1,4)$ induces the 2-partial permutation $(2,3)$, and we will denote the permutation by $(2,3 \mid 5,1,4)$ to emphasize the first 2 elements. If we push the third highest cell (cell 5) to the top, we get $(5,2 \mid 3,1,4)$.

Consider every $n$ cells as one logical digit, taking an alphabet size of $\left(\begin{array}{l}n \\ k\end{array}\right) k$ !. Suppose we have a cycle that traverses the $\left(\begin{array}{l}n \\ k\end{array}\right) k$ ! partial permutations, and increasing a logical digit by one corresponds to moving forward one step in the cycle. Then we can take a set of such logic digits, and increase some digits by a small amount at a time (e.g., floating code [3][4]), and construct a code for flash memory. The key result in this paper is a generalization of Gray codes from complete permutations [1] to $k$-partial permutations. In particular, we present Gray codes for arbitrary $k$-partial permutations that can be generated by push-to-the-top operations - providing efficient information update in flash memories.

Generating all partial permutations has been discussed before (e.g.,[5][6]). Our requirement of push-to-the-top operations is related to universal cycles of $k$-partial permutations [7][8][9][10]. A universal cycle of $k$-partial permutations is a sequence $\left(a_{1}, a_{2}, \ldots, a_{N}\right), N=\left(\begin{array}{l}n \\ k\end{array}\right) k !$, $a_{i} \in\{1,2, \ldots, n\}$, such that each $k$-partial permutation is represented by exactly one $\left(a_{i+1}, a_{i+2}, \ldots, a_{i+k}\right)$ for some $1 \leq i \leq N$. The index additions are computed modulo $N$. For a given $1 \leq i \leq N$, 
let $b_{1}, b_{2}, \ldots, b_{n-k} \in\{1,2, \ldots, n\}$ be distinct integers different from $\left\{a_{i+1}, \ldots, a_{i+k}\right\}$. Therefore, $P_{i}=\left(a_{i+k}, a_{i+k-1}, \ldots, a_{i+1} \mid b_{1}, b_{2}, \ldots, b_{n-k}\right)$ is a permutation. And let $b_{0}=a_{i+1}$. Then $a_{i+k+1}=b_{j}$ for some $0 \leq j \leq n-k$. Let $P_{i+1}=$ $\left(a_{i+k+1}, a_{i+k}, \ldots, a_{i+2} \mid b_{0}, \ldots, b_{j-1}, b_{j+1}, \ldots, b_{n-k}\right)$. From $P_{i}$ to $P_{i+1}$, we push $b_{j}=a_{i+k+1}$ to the top. Thus, traversing the $k$-partial permutations using push-to-thetop operation is identical to generating a universal cycle (see more details in [11]).

The existence of such universal cycles was proved in [8], however, the proof was nonconstructive. An explicit construction of a cycle for $k=n-1$ was given in [10]. But for other values of $k$, the construction is still an open problem. In this paper, we will provide constructions of a cycle for all $k \leq n-1$.

We will reduce the problem of generating $k$-partial permutations to two parts: generating all $\left(\begin{array}{l}n \\ k\end{array}\right)$ combinations and all permutations of $k-1$ elements. The latter is again the $(k-2)$-partial permutations out of $k-1$ elements.

Definitions and notations are introduced in Section II and the construction of Gray code for partial rank modulation is explained in Section III. Finally, concluding remarks are made in Section IV.

\section{Definitions AND Notations}

A $k$-partial permutation out of $n$ element is a $k$-tuple $Q=\left(p_{1}, p_{2}, \ldots, p_{k}\right)$ with distinct $k$ elements of a set of size $n$. The number of possible $k$-partial permutations is $\left(\begin{array}{l}n \\ k\end{array}\right) k$ !. The partial permutation is said to be induced from the permutation $P=\left(p_{1}, p_{2}, \ldots, p_{n}\right)$, for any ordering of $p_{k+1}, p_{k+2}, \ldots, p_{n}$. Sometimes we will denote this permutation by $P=\left(p_{1}, p_{2}, \ldots, p_{k} \mid p_{k+1}, p_{k+2}, \ldots, p_{n}\right)$ to emphasize the first $k$ elements.

A push-to-the-top operation, denoted by $t_{i}, \quad$ is a transition from $\left(p_{1}, p_{2}, \ldots, p_{n}\right)$ to $\left(p_{i}, p_{1}, p_{2}, \ldots, p_{i-1}, p_{i+1}, \ldots, p_{n}\right)$, for $2 \leq i \leq n$. Throughout this paper, left hand side is considered top, and right hand side is bottom. Notice that if $2 \leq i<k$, then after the operation $t_{i}$, there will be a charge gap between the $p_{i-1}$-th and the $p_{i+1}$-th cell. As information is stored in the highest $k$ cells, we want to keep these $k$ cells close in charge levels. Especially when large deflation happens, lower charge levels may decrease to none at all, and gap among the first $k$ cells may cause an error. Therefore, in our discussion, we only allow $t_{i}$, for $k \leq i \leq n$.

Consider a graph $G$, whose vertices are permutations of length $n$. There is a directed edge if there is a pushto-the-top operation $t_{i}$ from one vertex to another, for $k \leq i \leq n$. So each vertex in $G$ is restricted to $n-k+1$ outgoing edges. An $(n, k)$ Gray code for partial rank modulations is a cycle in $G$ whose vertices induce every $k$-partial permutation once and only once. A Gray code contains $N=\left(\begin{array}{l}n \\ k\end{array}\right) k$ ! permutations, and we denote them by $\mathbf{P}=\left(P_{1}, P_{2}, \ldots, P_{N}\right)$. The question is, does such a cycle exist? We will see in the paper that the answer is yes, and there are indeed a large number of such cycles.

Example 1. Figure 1(a) shows a $(4,2)$ Gray code. Only the top two digits in each permutation are of concern. And the push-to-the-top operations are indicated above each edge.

Define a partition on the partial permutations by the relation $\sim$, where $P_{1} \sim P_{2}$ if the first $k$ elements of $P_{2}$ is a cyclic shift of that of $P_{1}$. For example, if $k=3, n=6, E Q C=\{(1,3,6),(6,1,3),(3,6,1)\}$ is an equivalence class. $\sim$ is obviously an equivalence relation. If $P=\left(p_{1}, p_{2}, \ldots, p_{k}\right) \in E Q C$, and $p_{k}=$ $\max \left\{p_{1}, p_{2}, \ldots, p_{k}\right\}$, then we choose $P$ as the representative of the equivalence class EQC (unless mentioned otherwise). We will denote EQC by $\left(p_{1}, p_{2}, \ldots, p_{k}\right)$. In the previous example, $(1,3,6)$ is the representative and we will write $E Q C=(1,3,6)$. There are $\left(\begin{array}{l}n \\ k\end{array}\right)(k-1)$ ! equivalence classes.

\section{GRAY CODE FOR PARTIAL RANK MODULATION}

In this section, we present a construction of $(n, k)$ Gray code for partial rank modulation, for $1 \leq k \leq$ $n-1$. We first construct a tree that contains all the equivalence classes, and then use this tree to generate all the partial permutations.

Notice that starting from any partial permutation, if we do $(k-1)$ times the operation $t_{k}$, we can traverse an equivalence class. If we start from some partial permutation in equivalence class $E Q C_{1}$ and apply operations

$$
\underbrace{t_{k}, \ldots, t_{k}}_{a \text { times }}, t_{i}, \underbrace{t_{k}, \ldots, t_{k}}_{k-1 \text { times }}, t_{k+1}, \underbrace{t_{k}, \ldots, t_{k}}_{k-2-a \text { times }}
$$

for $0 \leq a \leq k-2$ and $i>k$, we can still traverse all members of $E Q C_{1}$. And if the partial permutation after $t_{i}$ belongs to $E Q C_{2}$, the above operations also traverse $E Q C_{2}$. In another word, inserting $E Q C_{2}$ does not affect the completion of $E Q C_{1}$. We call the above operation insertion. In addition, if $a=k-1$ in the above operation sequence, we omit the last operations $t_{k+1}$ and $(k-2-a)$ times of $t_{k}$. And we traverse $E Q C_{1}$, followed by traversing $E Q C_{2}$. We consider this case as insertion, too. The permutations circled by dashed line in Figure 1(a) illustrates this notion, where $E Q C_{1}=(2,4)$ is inserted by $E Q C_{2}=(2,3)$.

We will draw insertions in an insertion tree. The nodes of the tree are equivalence classes. An edge means the child is inserted in the parent. Notice the parent and the child differ in only one digit for their representatives. We can insert at most $k$ classes to $E Q C_{1}$, each changing distinct digit of $E Q C_{1}$. The cycle in Figure 1(a) is generated by the insertion tree in Figure 1(b). The following lemma is similar to the cycle merging technique in [9], however, the ordering of the lower $n-k$ elements matters in our case. 


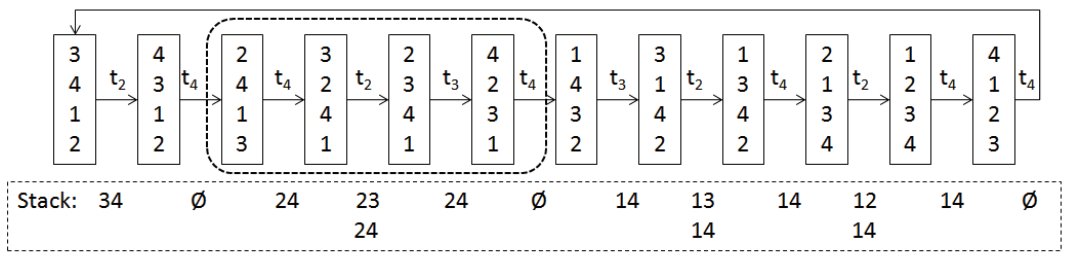

(a)

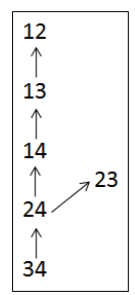

(b)

Fig. 1. (a) A cycle for 2-partial permutations out of 4. The corresponding stack storing equivalence classes are shown in the lower dashed box. (b) The insertion tree generating the cycle in (a).

Lemma 2. Any insertion tree of $k$ elements out of $n$ that contains each equivalence class once and only once will generate an $(n, k)$ Gray code. Such an insertion tree is called a generating tree.

Proof: We will first prove that a generating tree will induce a path of all $k$-partial permutations.

Claim: an insertion tree will induce a path of all members of the equivalence classes of the tree (possibly with duplicates if the tree has duplicated nodes). Moreover, the first permutation of the path can be $\left(p_{1}, p_{2}, \ldots, p_{k} \mid p_{k+1}, \ldots, p_{n}\right)$, for any $\left(p_{1}, p_{2}, \ldots, p_{k}\right)$ in the equivalence class of the root, and any $X=$ $\left(p_{k+1}, \ldots, p_{n}\right)$ as the lower $n-k$ elements.

Use induction on the depth. Base case is one single node, which is true by doing $k-1$ times $t_{k}$. For a tree with depth of $D$, the sub-trees of the root have depth of at most $D-1$ and, by induction, generate paths of all members of their equivalence classes. Let the root be $\left(p_{1}, p_{2}, \ldots, p_{k}\right)$ ( $p_{k}$ is not necessarily the largest). For one sub-tree, suppose its root is $\left(p_{1}, p_{2}, \ldots, p_{i-1}, p_{i}^{\prime}, p_{i+1}, \ldots, p_{k}\right)$, then we can construct a path as follows: $\left(p_{1}, p_{2}, \ldots, p_{k} \mid X_{1}\right) \stackrel{t_{k}}{\rightarrow}$ $\ldots \stackrel{t_{k}}{\rightarrow} \quad\left(p_{i+1}, \ldots, p_{k}, p_{1}, \ldots, p_{i} \mid X_{1}\right) \quad \stackrel{\text { push } p_{i}^{\prime}}{\rightarrow}$ $\left[\left(p_{i}^{\prime}, p_{i+1}, \ldots, p_{k}, p_{1}, \ldots, p_{i-1} \mid X_{2}\right) \quad \rightarrow \quad \cdots \rightarrow\right.$ $\left.\left(p_{i+1}, \ldots, p_{k}, p_{1}, \ldots, p_{i-1}, p_{i}^{\prime} \mid X_{3}\right)\right] \quad \stackrel{\text { push } p}{\longrightarrow}$ $\left(p_{i}, p_{i+1}, \ldots, p_{k}, p_{1}, \ldots, p_{i-1} \mid X_{4}\right) \quad \stackrel{t_{k}}{\rightarrow} \quad \ldots \quad \stackrel{t_{k}}{\rightarrow}$ $\left(p_{2}, \ldots, p_{k}, p_{1} \mid X_{4}\right)$, for some $X_{1}, X_{2}, X_{3}, X_{4}$ as the lower elements of the permutations. The sub-path in the square brackets corresponds to the sub-tree. If $i=1$, the part after the square brackets is omitted. This path goes through the equivalence classes in the sub-tree and the root completely. Similarly, we can insert other sub-paths into this path. So the claim is true.

Now as a generating tree contains each equivalence class exactly once, we have a path passing every partial permutation exactly once. Next, noticing that such a path satisfies the condition in Lemma 2 in [11], an $(n, k)$ Gray code (a cycle) can be generated.

This Gray code in Lemma 2 works like a stack. That is, when we go to the current node $m$, put it in the stack. If every element in the equivalence class of $m$ is passed through, remove $m$ from the stack. Next, go to a child of $m$, and treat the child as the current node. If the current node has been traversed, and has no children, then pop a node from the stack and go to that node. For example, in Figure 1(a), the stack after each partial permutation is written in the lower dashed box.

We will give a special class of generating trees based on combinations, where the elements are ordered increasingly. Let $\left(c_{1}, c_{2}, \ldots, c_{n}\right)$ be a vector with distinct values and $c_{p_{1}}>c_{p_{2}}>\cdots>c_{p_{n}}$, then define its reversed rank as $\left(p_{n}, p_{n-1}, \ldots, p_{1}\right)$. Suppose we have an insertion tree $T_{1}$ that contains each combination of $k$ out of $n$ once, and denote a node in $T_{1}$ by $\left(p_{1}, p_{2}, \ldots, p_{k}\right)$, $p_{1}<p_{2}<\cdots<p_{k}$. Suppose the root of the tree is $(n-k+1, n-k+2, \ldots, n)$. Define $\sigma_{1}$ to be the identity permutation on $\{1,2, \ldots, k-1\}$. Let $\sigma_{2}$ be a permutation of $\{1,2, \ldots, k-1\}$. And for an integer $x$, use $\sigma_{2}+x$ to denote the sequence $\left(\sigma_{2}(1)+x, \sigma_{2}(2)+\right.$ $\left.x, \ldots, \sigma_{2}(k-1)+x\right)$. If we can transit from a node in $T_{1}$ to the node $\left(\sigma_{2}+(n-k), n\right)$, then by the same structure as $T_{1}$, except that we change each $\left(p_{1}, p_{2}, \ldots, p_{k}\right)$ in $T_{1}$ to $\left(p_{\sigma_{2}(1)}, p_{\sigma_{2}(2)}, \ldots, p_{\sigma_{2}(k-1)}, p_{k}\right)$ in $T_{2}$, we get another insertion tree $T_{2}$ and the dual (the inverse permutation) of the reversed rank of each node in $T_{2}$ is $\left(\sigma_{2}, k\right)$. After that, we transit from $T_{2}$ to $T_{3}$ with permutation $\sigma_{3}$, and each node in $T_{1}$ is replaced by $\left(p_{\sigma_{3}(1)}, p_{\sigma_{3}(2)}, \ldots, p_{\sigma_{3}(k-1)}, p_{k}\right)$, and so on.

Theorem 3. If there is an insertion tree $T_{1}$ that contains every combination of $k$ out of $n$ once, and a sequence of valid transitions from previous trees (as described above), $\sigma_{1}, \sigma_{2}, \ldots, \sigma_{(k-1) !}$, traversing all permutations on $\{1,2, \ldots, k-1\}$, then $T_{1} \rightarrow T_{2} \rightarrow \cdots \rightarrow T_{(k-1) !}$ is a generating tree of $k$-partial permutations.

Proof: Recall that we represent the equivalence classes with $\left(p_{1}, \ldots, p_{k}\right)$ where $p_{k}=\max \left\{p_{1}, \ldots, p_{k}\right\}$. For any $p_{1}<p_{2}<\cdots<p_{k}$, and any equivalence class $\left(p_{\sigma(1)}, p_{\sigma(2)}, \ldots, p_{\sigma(k-1)}, p_{k}\right)$, there exists exactly one $1 \leq i \leq(k-1)$ !, such that $\sigma=\sigma_{i}$ and this equivalence class appears exactly once in the tree $T_{i}$.

Now the problem of finding Gray code for partial rank modulation reduces to finding $T_{1}$ and $\sigma_{1}, \sigma_{2}, \ldots, \sigma_{(k-1) !}$.

Construction 4. (Construction of $\left.T_{1}\right)$ The root $(n-$ $k+1, n-k+2, \ldots, n)$ has one child $(n-k, n-$ $k+2, \ldots, n)$, and the node $(1,2, \ldots, k)$ has a parent 
$(1,2, \ldots, k-1, k+1)$. Starting from the root, and following the connection rules below for all the other nodes, we will get a $T_{1}$.

1) A node $\left(p_{1}, p_{2}, \ldots, p_{k-n+t-1}, t, t+1, \ldots, n\right)$, for $1 \leq p_{k-n+t-1}<t-1$ and $n-k+2 \leq t \leq n$, is connected to the at most 3 nodes:

Parent $\left(p_{1}, p_{2}, \ldots, p_{k-n+t-1}+1, t, t+1, \ldots, n\right)$

Child $_{1}\left(p_{1}, p_{2}, \ldots, p_{k-n+t-1}-1, t, t+1, \ldots, n\right)$ if $p_{k-n+t-1}-1>p_{k-n+t-2} \geq 1$

Child $_{2}\left(p_{1}, p_{2}, \ldots, p_{k-n+t-1}, t-1, t+1, \ldots, n\right)$

2) Otherwise, a node $\left(p_{1}, p_{2}, \ldots, p_{k}\right)$ is connected to at most two nodes:

$$
\begin{aligned}
& \text { Parent }\left(p_{1}, p_{2}, \ldots, p_{k}+1\right) \\
& \text { Child }\left(p_{1}, p_{2}, \ldots, p_{k}-1\right) \text { if } p_{k}-1>p_{k-1}
\end{aligned}
$$

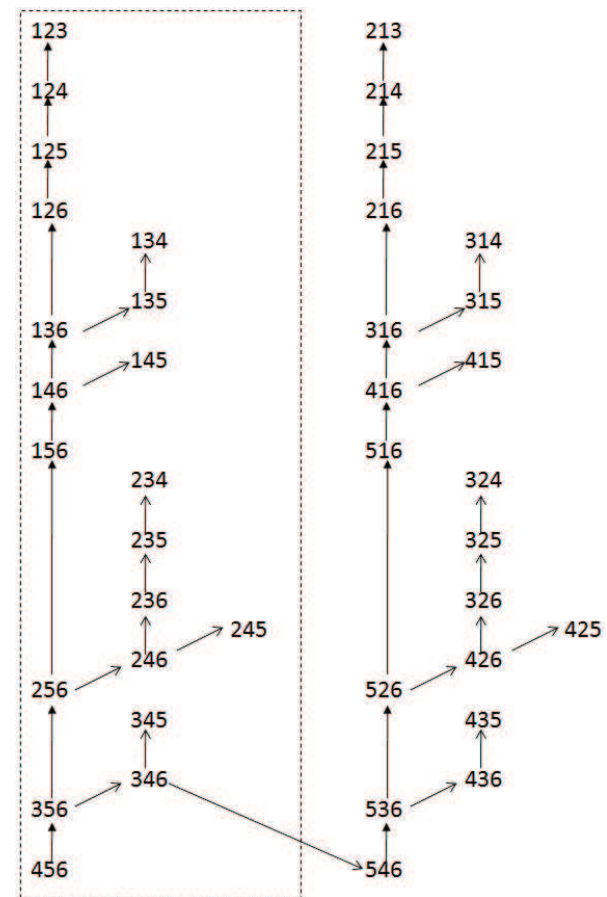

Fig. 2. A generating tree of 6 out of 3 based on Construction 4 and cycle $12 \stackrel{t_{2}}{\rightarrow} 21 \stackrel{t_{2}}{\rightarrow} 12$

Figure 1(b) is one example of Construction 4. Another example of 6 out of 3 is shown in Figure 2. $T_{1}$ is the left part of the tree in dashed line. $T_{1}$ is similar to listing combinations lexicographically.

Theorem 5. Construction 4 forms a tree $T_{1}$ that contains every combination of $k$ out of $n$.

Proof: The construction already includes every combination. So we need to prove that $T_{1}$ is a connected graph with no cycles.

Starting from any node in $T_{1}$, and tracing back the parent, is equivalent to increasing the $k$-th digit to the maximum, and then increasing the $(k-1)$-th digit to the maximum, and so on. All of these backward paths will end at $(n-k+1, n-k+2, \ldots, n)$. Therefore, graph $T_{1}$ is connected. Moreover, as a node is always smaller than its parent in lexicographical order, there is no cycle in $T_{1}$. Hence $T_{1}$ is a tree.

Another possible $T_{1}$ is a single line. There are several different constructions in the literature. For example, homogeneous scheme and near-perfect scheme in [12].

For construction of $\sigma_{1}, \ldots, \sigma_{(k-1) !}$, we will assume $k \geq 3$, because otherwise only $T_{1}$ itself is the generating tree. We first find which transitions are allowed from sub-tree $T_{t}$ to $T_{t+1}$. Consider the node in $T_{t}, a=\left(p_{1}, p_{2}, \ldots, p_{i-1}, n-k, p_{i+1}, \ldots, p_{k-1}, n\right)$, where $\left\{p_{1}, \ldots, p_{i-1}, p_{i+1}, \ldots, p_{k-1}\right\}=\{n-k+$ $1, n-k+2, \ldots, n-1\} \backslash\{y\}$ for some $n-k+$ $1<y \leq n-1$. This node exists since $n \geq$ $k+1$. We can now define a child of $a$ as $b=$ $\left(p_{1}, p_{2}, \ldots, p_{i-1}, y, p_{i+1}, \ldots, p_{k-1}, n\right)$. Let $x=y-$ $n+k$ and $b$ be the root of $T_{t+1}$. Since $\left(\sigma_{t}, k\right)$ is dual of reversed rank of $a$ and $\left(\sigma_{t+1}, k\right)$ is dual of reversed rank of $b$, we can see that $\sigma_{t+1}=\alpha_{x} \circ \sigma_{t}$, where $\alpha_{x}=(x, 1,2, \ldots, x-1, x+1, \ldots, k-1)$. Here $\circ$ is the composition of permutations and is computed from right to left. It can be seen that the child of $a$ in Construction 4 changes a different digit of $a$, compared to $b$. Hence, $\alpha_{x}$ is a valid transition from tree $T_{t}$ to $T_{t+1}$.

Permuted by $\alpha_{x}$, the lowest position in $\sigma_{t}$ becomes the $x$-th lowest position in $\sigma_{t+1}$. In another word, from the dual of $\sigma_{t+1}$ to the dual of $\sigma_{t}$, we push the $x$-th element to the top.

Construction 6. (Construction of $\sigma_{1}, \ldots, \sigma_{(k-1) !}$ ) Suppose $\mathcal{C}$ is a cycle of permutations of $\{1,2, \ldots, k-1\}$. First take the dual of each permutation in $\mathcal{C}$. Then replace each push-to-the-top operation $t_{x}$ with $\alpha_{x}$ and reverse the edge direction. And thus we will get a cycle of $\sigma_{1}, \sigma_{2}, \ldots, \sigma_{(k-1) !}$, generated by operations $\alpha_{x}, 1<x \leq$ $k-1$. Starting from the identity permutation $\sigma_{1}$, and ig noring the last edge, we get a path of $\sigma_{1}, \sigma_{2}, \ldots, \sigma_{(k-1) !}$.

$\sigma_{1}, \sigma_{2}, \ldots, \sigma_{(k-1) !}$ can be easily generated once we have recursively generated $(k-2)$-partial permutations out of $k-1$ (or permutations on $k-1$ elements) using the constructions above. We can also use the cycle of permutations in [1][10]. For example, if $k=4$, then Construction 4 will form a cycle $\mathcal{C}$ on 3 elements using the operation sequence $t_{2}, t_{3}, t_{3}, t_{2}, t_{3}, t_{3}$. And the push-tothe-bottom sequence is $231 \stackrel{t_{2}}{\longrightarrow} 321 \stackrel{t_{3}}{\longrightarrow} 132 \stackrel{t_{3}}{\longrightarrow} 213 \stackrel{t_{2}}{\longrightarrow}$ $123 \stackrel{t_{3}}{\rightarrow} 312 \stackrel{t_{3}}{\rightarrow} 231$. The corresponding sigma cycle is $312 \stackrel{\alpha_{2}}{\longleftarrow} 321 \stackrel{\alpha_{3}}{\longleftarrow} 132 \stackrel{\alpha_{3}}{\longleftarrow} 213 \stackrel{\alpha_{2}}{\longleftarrow} 123\left(\stackrel{\alpha_{3}}{\longleftarrow}\right) 231 \stackrel{\alpha_{3}}{\longleftarrow} 312$. Deleting the arrow in brackets, we get a path.

Having constructed $T_{1}$ and $\sigma_{1}, \ldots, \sigma_{(k-1)}$ ! we are able to draw a generating tree. Figure 2 shows a generating tree of 6 out of 3 . The sequence $\sigma_{1}, \sigma_{2}$ is $12 \stackrel{\alpha_{2}}{\rightarrow} 21$, and the transition node from $T_{1}$ to $T_{2}$ is $(3,4,6)$.

Knowing a generating tree and the current partial permutation, how shall we decide the operation for next 
step? Define $w_{m}$ to be the position of the newly changed digit from the parent to the node $m$ (counted from top to bottom). Let $m=\left(p_{1}, \ldots, p_{k}\right)$, then its parent defers with $m$ only at $p_{w_{m}}$. For the root, $w_{\text {root }}$ can be any integer between 1 and $k$. But we will assign $w_{\text {root }}$ as $k$. For example, in Figure 2, $(1,2,4)$ is the parent of the node $m=(1,2,3)$, and the 3rd digit is changed from 4 to 3 , so $w_{m}=3, p_{w_{m}}=p_{3}=3$. The following algorithm recursively computes $\alpha_{x}$ and the next permutation:

\section{Algorithm 7. $(G(n, k))$}

Given a permutation, take the top $k$ elements $\left(q_{1}, q_{2}, \ldots, q_{k}\right)$, and suppose $q_{l}=\max _{i=1}^{k} q_{i}$. Let $j-1$ be the number of elements appearing after $q_{l}$. Order the top $k$ elements increasingly as $m=\left(p_{1}, p_{2}, \ldots, p_{k}\right)$.

1 If $m=\left(p_{1}, p_{2}, \ldots, p_{i-1}, n-k, p_{i+1}, \ldots, p_{k-1}, n\right)$, where $\left\{p_{1}, \ldots, p_{i-1}, p_{i+1}, \ldots, p_{k-1}\right\}=\{n-k+$ $1, n-k+2, \ldots, n-1\} \backslash\{y\}$ for some $n-k+$ $1<y \leq n-1$, and if $j-1 \equiv k \bmod k$, compute $\sigma$ and $\alpha_{x} . \sigma$ is the dual of the reversed rank of $\left(q_{l+1}, q_{l+2}, \ldots, q_{k}, q_{1}, \ldots, q_{l-1}\right)$, and $\alpha_{x}$ is obtained recursively by the reverse algorithm of $G(k-1, k-2)$ on dual of $\sigma$. If $x=y-n+k$, push $x$ to the top (Transit to the next sub-tree).

2 Else, if $m=(n-k+1, n-k+2, \ldots, n)$ and $j-$ $1 \equiv k \bmod k$, then push $n-k$ to the top (Transit to the previous sub-tree).

3 Else, first compute parameters: if $m=$ $\left(p_{1}, p_{2}, \ldots, p_{k-n+t-1}, t, t+1, \ldots, n\right), \quad$ for $1 \leq p_{k-n+t-1}<t-1$ and $n-k+2 \leq t \leq n$, then $w_{m}=k-n+t-1, w_{y}=k-n+t-1$ (only if $p_{k-n+t-1}-1>p_{k-n+t-2} \geq 1$ ) and $w_{y^{\prime}}=k-n+t$, and $p_{w_{y}}^{(y)}$ is $p_{k-n+t-1}-1$ (only if $p_{k-n+t-1}-1>p_{k-n+t-2} \geq 1$ ), and $p_{w_{y^{\prime}}}^{\left(y^{\prime}\right)}=t-1$, respectively; else, $w_{m}=w_{y}=k$, and $p_{w_{y}}^{(y)}=p_{k}-1$.

- If $p_{k-1}+1=p_{k} \neq n$ ( $m$ has no children): if $j \equiv w_{m}+1 \bmod k$, then push $p_{k}+1$ to the top; else, do $t_{k}$.

- Else ( $m$ has children): if $j \equiv w_{y}+1$ (or $\left.j \equiv w_{y^{\prime}}+1\right) \bmod k$ for any child, push $p_{w_{y}}^{(y)}$ (or $p_{w_{y^{\prime}}}^{\left(y^{\prime}\right)}$ ) to the top; else if $j \equiv w_{m}+1$ $\bmod k$, then push $t-1$ to the top if $m=$ $\left(p_{1}, p_{2}, \ldots, p_{k-n+t-1}, t, t+1, \ldots, n\right)$ for $1 \leq$ $p_{k-n+t-1}<t-1$, and $n-k+2 \leq t \leq n$, otherwise push $n$ to the top; else do $t_{k}$.

Figure 1(a) is an example of the above algorithm with $n=4, k=2$. For instance, if the current permutation is $(1,4 \mid 2,3)$, then $j-1=0$ and $m=\left(p_{1}, p_{2}\right)=(1,4)$. $\sigma$ is identity. $t=p_{2}=4, w_{m}=k-n+t-1=1$, and $w_{y^{\prime}}=k-n+t=2$. As $j=w_{y^{\prime}}+1$, We push $p_{w_{y^{\prime}}}^{\left(y^{\prime}\right)}=t-1=3$ to the top. The next permutation is $(3,1 \mid 4,2)$.
Even though the algorithm $G(n, k)$ is recursive, we can show the number of recursions is less than 1 on average [11]. Hence, the average computational complexity of $G(n, k)$ is only dependent on the non-recursive operations. In particular, dependent on ordering the top $k$ elements increasingly as $m$. Thus, the complexity for Algorithm 7 is $O(k \log k)$.

\section{CONCLUSION}

In this paper we proposed a generalization of rank modulation - $k$-partial rank modulation - for flash memories. This coding scheme allows less decoding cost while sacrificing some information capacity. We presented a Gray code that traverses all $k$-partial permutations out of $n$ elements, using push-to-the-top write operations, which is designed specifically for flash cells. The permutation cycle is reduced first to a generating tree with cyclic shift classes as nodes. In the generating tree, adjacent nodes differ in one digit. And then it is further simplified to combination generation and permutation generation. Each step in the constructed cycle can be determined solely by the current permutation.

Several problems are still open in this topic. For instance, how to construct insertion trees with minimum, maximum, or evenly distributed gap in the lower $n-k$ cells. Also, whether it is possible to use the lower cells as redundancy to protect the top $k$ cells against errors.

\section{ACKNOWLEDGMENT}

This work was supported in part by the NSF Grant ECCS-0802107 and in part by an NSF-NRI award.

\section{REFERENCES}

[1] A. Jiang, R. Mateescu, M. Schwartz, and J. Bruck. Rank modulation for flash memories. Information Theory, IEEE Transactions on, 55(6):2659 -2673, June 2009.

[2] J. E. Brewer and M. Gill. Nonvolatile memory technologies with emphasis on flash. Wiley-IEEE, 2007.

[3] A. Jiang, V. Bohossian, and J. Bruck. Floating codes for joint information storage in write asymmetric memories. Proc. IEEE ISIT, 2007.

[4] M. Mitzenmacher, Z. Liu, and H. Finucane. Designing floating codes for expected performance. Proc. of the Forty-Sixth Annual Allerton Conference, 2008.

[5] G. Ehrlich. Loopless algorithms for generating permutations, combinations, and other combinatorial configurations. Journal of the ACM (JACM), 20(3):500-513, 1973.

[6] N. Dershowitz. A simplified loop-free algorithm for generating permutations. BIT Numerical Mathematics, 15(2):158-164, 1975.

[7] R. Graham F. Chung, P. Diaconis. Universal cycles for combinatorial structures. Discrete Mathematics, 110(1-3):43-59, 1992.

[8] B. W. Jackson. Universal cycles of k-subsets and k-permutations. Discrete mathematics, 117(1-3):141-150, 1993.

[9] J.R. Johnson. Universal cycles for permutations. Discrete Mathematics (in press), 2008.

[10] F. Ruskey and A. Williams. An explicit universal cycle for the (n1)-permutations of an n-set. In Talk at Napier Workshop. Citeseer, 2008.

[11] Z. Wang and J. Bruck. Partial rank modulation for flash memories. available at $h t t p: / / w w w . p a r a d i s e . c a l t e c h . e d u / e t r . h t m l$.

[12] D. E. Knuth. The art of computer programming, Volume 4, Fascicle 3. Addison-Wesley, 2005. 